\title{
Role of ASC in hypoxia-mediated cell death in pancreatic cancer
}

\author{
TAKAO OHTSUKA ${ }^{1}$, MAYUMI MITSUNO ${ }^{1}$, YOSHIHIKO KITAJIMA ${ }^{1}$, TAKAO IDE ${ }^{1,2}$, \\ SAM W. LEE ${ }^{2}$ and KOHJI MIYAZAKI ${ }^{1}$ \\ ${ }^{1}$ Department of Surgery, Saga University Faculty of Medicine, Saga, Japan; ${ }^{2}$ Cutaneous Biology Research Center, \\ Massachusetts General Hospital and Harvard Medical School, Boston, MA, USA
}

Received May 22, 2008; Accepted September 19, 2008

DOI: $10.3892 / \mathrm{mmr} 00000035$

\begin{abstract}
ASC, an apoptosis-associated speck-like protein, can regulate apoptosis in response to various types of cell death stimuli. In this study, we investigated the role of ASC in hypoxia-mediated cell death in pancreatic cancer. ASC was inducible under a $1 \% \mathrm{O}_{2}$ hypoxic condition in pancreatic cancer cells, which was HIF1 $\alpha$-dependent but p53-independent. Two representative chemotherapeutic agents for pancreatic cancer, 5-fluorouracil and gemcitabine, promoted cell death in a p53-dependent manner; however, $1 \%$ hypoxia caused chemoresistance to these drugs. Western blot analysis of this condition showed that expression of Bax, a pro-apoptotic gene, decreased, while the anti-apoptotic genes IAP-2 and survivin increased. These results suggest that, although hypoxia induces both pro-apoptotic and anti-apoptotic genes, the total balance seems to be anti-apoptotic dominant, which might explain chemoresistance in pancreatic cancer. To overcome this antiapoptotic dominant condition, we infected adenovirusexpressing ASC into pancreatic cancer cells. The expressed ASC induced cell death even under $20 \%$ normoxia, and was enhanced by hypoxia. Our data demonstrate the possible mechanism of chemoresistance under hypoxia in pancreatic cancer cells, thereby suggesting the potential use of ASC as a new treatment strategy for pancreatic cancer.
\end{abstract}

\section{Introduction}

Despite recent advances in surgical techniques and the discovery of new chemotherapeutic agents, pancreatic cancer remains difficult to cure (1). One of the reasons for its poor patient prognosis is considered to be the hypoxic conditions associated with this disease due to its hypovascularity; many previous studies have demonstrated that hypoxia causes chemoresistance and accelerates tumor progression, invasiveness and metastasis in pancreatic cancer (2-8).

Correspondence to: Dr Takao Ohtsuka, Department of Surgery, Saga University Faculty of Medicine, 5-1-1 Nabeshima, Saga 849-8501, Japan

E-mail: ootsuka4@cc.saga-u.ac.jp

Key words: hypoxia, pancreatic cancer, ASC
ASC, an apoptosis-associated speck-like protein, is a unique protein which consists of two protein-protein interaction domains, an amino-terminal pyrin domain and a caspase recruitment domain in the carboxyl terminal (9). We previously demonstrated that ASC is a target gene of p53 and regulates the p53-Bax mitochondrial apoptotic pathway induced by many chemotherapeutic drugs $(10,11)$. As well, we showed that ASC can regulate the p53-independent apoptotic pathway by such agents as tumor necrosis factor (TNF)- $\alpha$ or indomethacin (10). Others have shown that ASC affects nuclear factor $\kappa-B$ (NF-kB) function through its binding to IKK in response to pro-inflammatory stimulation (12). Therefore, ASC can regulate cell death signaling in response to various types of stimuli, and it is possible that ASC may be a regulator of hypoxia-mediated cell death signaling as well. In this study, we investigated the role of ASC in hypoxia-mediated cell death using pancreatic cancer cells.

\section{Materials and methods}

Cell lines and constructs. Seven pancreatic cancer cell lines, PK-1, KP-4, PK-8, PK-9, PK-59, Miapaka and KLM-1, were purchased from the Institute of Development, Aging and Cancer, Tohoku University (Sendai, Japan) and from the Riken Cell Bank (Ibaragi, Japan). The cells were cultured in RPMI-1640 medium (Sigma) containing 10\% fetal bovine serum (Sigma) and $100 \mu \mathrm{g} / \mathrm{ml} \mathrm{kanamycin} \mathrm{(Meiji,} \mathrm{Tokyo,}$ Japan), and were incubated at $37^{\circ} \mathrm{C}$ in a humidified atmosphere containing $20 \% \mathrm{O}_{2}$ and $5 \% \mathrm{CO}_{2}$ in air. Thereafter, some cells were moved to a hypoxic chamber (Astec, Fukuoka, Japan) containing $1 \% \mathrm{O}_{2}, 5 \% \mathrm{CO}_{2}$ and $94 \% \mathrm{~N}_{2}$ at $37^{\circ} \mathrm{C}$. Recombinant adenovirus-expressing myc-tagged ASC (Ad-ASC) and green fluorescent protein (Ad-GFP) as a control were previously described (10). Sense and antisense oligonucleotides corresponding to the following cDNA sequences were purchased from iGene (Ibaragi, Japan): CCAGCAGACUCAAAUACAAGAACCUAG for hypoxiainducible factor- $1 \alpha(\mathrm{HIF}-1 \alpha)$ and GAGAGCUGAUUUACG GAUGUAGAAGAG for scramble (control). The cells were grown to $50 \%$ confluency in a 6-well plate and infected with adenovirus at a multiplicity of infection of 30, or transfected with small interference RNA (siRNA) using a Microporator (Digital Bio Technology Co., Suwon, Korea) according to the manufacturer's instructions in the presence of siRNA. Regarding drug treatment, the cells were exposed to 5-fluorouracil 


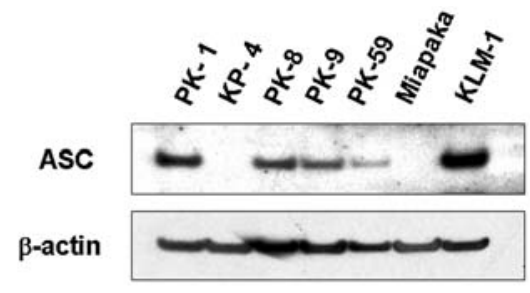

Figure 1. The expression of the protein level of ASC in pancreatic cancer cells. Seven pancreatic cancer cell lines were lysed in lysis buffer and subjected to Western blot analysis using antibodies against ASC and ß-actin (control).

(5-FU, Sigma) at a concentration of $600 \mathrm{nM}$ or gemcitabine (GEM, Eli Lilly) at $1 \mu \mathrm{M}$ for $24 \mathrm{~h}$.

Western blot analysis. Cells were lysed in lysis buffer (20 mM Tris, pH 7.4, 5 mM EDTA, $10 \mathrm{mM} \mathrm{Na}_{4} \mathrm{P}_{2} \mathrm{O}_{7}, 100 \mathrm{mM}$ $\mathrm{NaF}, 2 \mathrm{mM} \mathrm{Na} \mathrm{VO}_{4}, 1 \% \mathrm{NP}-40$ and $1 \mathrm{mM}$ PMSF) including Protease Inhibitor Cocktail (1:200 dilution, Sigma). Equal amounts of total cellular proteins per sample were subjected to SDS-PAGE on a 10\% Bis-Tris gel (Invitrogen) and then transferred to a nitrocellulose membrane (Invitrogen). Primary antibodies for immunoblotting included anti-ASC (Chemicon), -p53 (DO-1; Santa Cruz), -HIF-1 $\alpha$ (R2; Novus), -IAP-2 (H-85, Santa Cruz), -survivin (FL142, Santa Cruz), -Bax (N-20, Santa Cruz) and -ß-actin (Sigma). Secondary antibodies were goat anti-rabbit and rabbit anti-mouse conjugated with horseradish peroxidase (Santa Cruz). Bands were detected using the ECL chemiluminescence detection method (Amersham).

Cell death analysis (Trypan blue exclusion test). Cells were pelleted and washed with phosphate-buffered saline. Trypan blue (Gibco) was added to the cell pellets, and stained cells were counted as dead. Experiments were repeated 3 times, and values were expressed as the mean \pm standard deviation.

\section{Results}

Expression of ASC in pancreatic cancer cells. Seven pancreatic cancer cell lines were lysed in lysis buffer and subsequently subjected to Western blot analysis. Fig. 1 shows that ASC expression was observed in $5 / 7$ cell lines $(71 \%)$. Of these,
PK-1 was mainly used in our experiments since ASC, wildtype p53, HIF-1 $\alpha$ and other apoptosis-associated genes such as Bax, IAP-2 and survivin were all well preserved.

ASC induction under hypoxia. PK-1 and PK-8 (p53-null) were incubated in $1 \% \mathrm{O}_{2}$ and collected at the indicated times. Fig. 2A shows ASC induction under hypoxia in PK-1 cells. HIF-1 $\alpha$ was also induced by hypoxia, while the protein level of p53 was no different from the level observed under normoxia. When using p53-null PK-8 cells, ASC induction was also noted, with a parallel change in HIF- $1 \alpha$ and its target gene IAP-2 (Fig. 2B). To ascertain whether ASC induction was HIF- $1 \alpha$-dependent or not, siRNA against HIF- $1 \alpha$ was transfected into PK-1 cells and incubated under hypoxia. This siRNA construct almost totally suppressed the expression of HIF- $1 \alpha$ under hypoxia, and the expression levels of ASC and IAP-2 also decreased in comparison to untransfected cells under hypoxia (Fig. 2C). These data suggest that ASC induction under hypoxia is p53-independent but HIF-1 $\alpha$ dependent.

Effect of hypoxia on chemosensitivity. To determine the effect of hypoxia on chemosensitivity in pancreatic cancer cells, PK-1 cells were treated by two representative chemotherapeutic agents for pancreatic cancer, GEM and 5-FU, with or without $1 \% \mathrm{O}_{2}$ hypoxia for $24 \mathrm{~h}$. Fig. 3A shows that GEM induced cell death through the $\mathrm{p} 53-\mathrm{Bax}$ pathway, and that a $1 \% \mathrm{O}_{2}$ hypoxic condition inhibited such chemosensitivity. Notably, Western blot analysis demonstrated that hypoxia suppressed the expression level of Bax, known as a key regulator of the p53mitochondrial cell death signaling pathway, but increased the protein levels of anti-apoptotic molecules such as IAP-2 and survivin (Fig. 3B). The same phenomenon was observed when applying 5-FU (Fig. 3C and D). These results indicate that although hypoxia induces both a pro-apoptotic gene (ASC induction) and an anti-apoptotic condition (Bax repression and inductions of IAP-2 and survivin), the total balance seems to be anti-apoptotic dominant.

Effect of expressed ASC on hypoxia-mediated cell death. PK-1 cells were infected with Ad-ASC or Ad-GFP (as a control), and were incubated $24 \mathrm{~h}$ after infection with or without hypoxia, then collected at the indicated times to determine cell death
A.

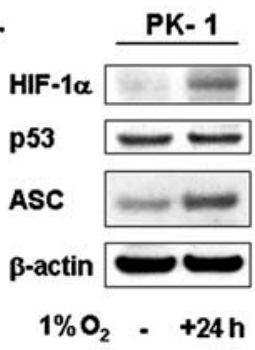

B.

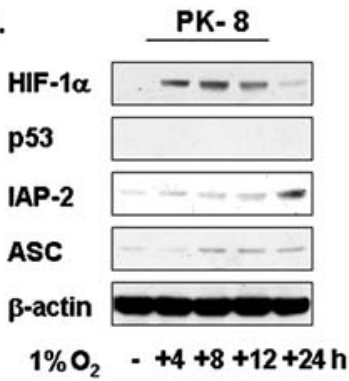

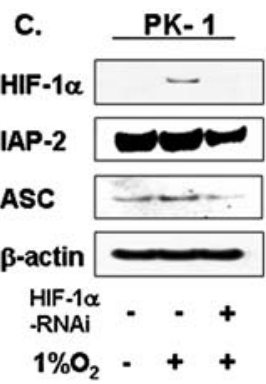

Figure 2. (A) The induction of ASC under hypoxia. PK-1 cells were incubated in $1 \% \mathrm{O}_{2}$ for $24 \mathrm{~h}$ and subjected to Western blot analysis using antibodies against HIF-1 $\alpha$, p53, ASC and B-actin. (B) The p53-independent induction of ASC under hypoxia. PK-8 cells (p53-null) were incubated in $1 \%$ O $\mathrm{O}_{2}$ and collected at the indicated times. Next, the cells were subjected to Western blot analysis using anti-HIF-1 $\alpha$, -p53, -ASC, -IAP-2 and - $\beta$-actin antibodies. (C) ASC induction under hypoxia is HIF-1 $\alpha$-dependent. PK-1 cells were transfected with siRNA against HIF-1 $\alpha$ or scramble siRNA (control). Twenty-four hours after transfection, the cells were incubated under $20 \% \mathrm{O}_{2}$ normoxia or $1 \% \mathrm{O}_{2}$ hypoxia. Next, the cells were collected and subjected to Western blot analysis. 
A.

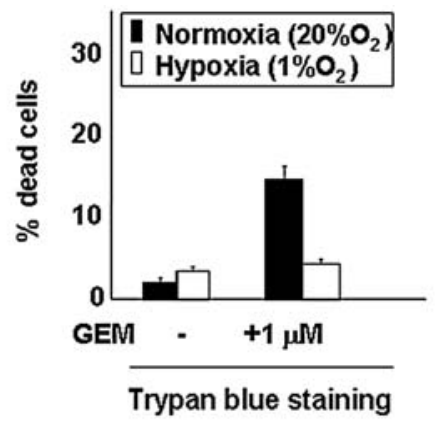

c.

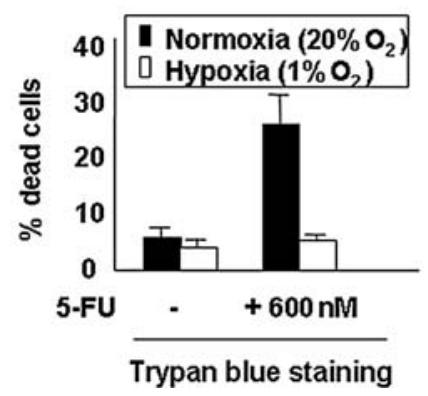

B.

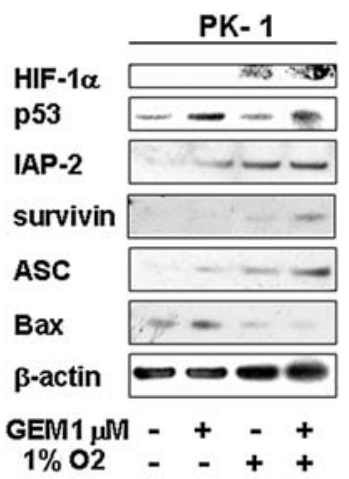

D.

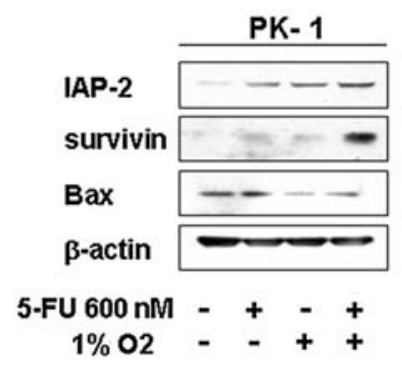

Figure 3. Hypoxia induces chemoresistance. (A) PK-1 cells were treated with $1 \mu \mathrm{M}$ of gemcitabine (GEM) with or without hypoxia for $24 \mathrm{~h}$. (B) Next the cells were subjected to Western blot analysis and Trypan blue exclusion analyses. The primary antibodies were anti-HIF-1 $\alpha$, -p53, -IAP-2, -survivin, -ASC, -Bax and -B-actin. (C and D) PK-1 cells were treated with $600 \mathrm{nM}$ of 5-fluorouracil (5-FU) with or without hypoxia for $24 \mathrm{~h}$. Thereafter, the cells were subjected to Trypan blue staining analyses (C) and Western blot analysis (D).
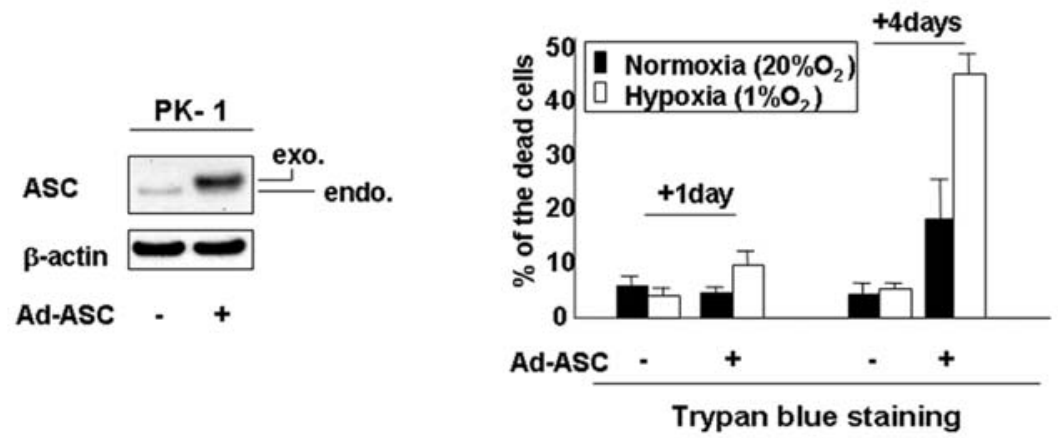

Figure 4. Hypoxia enhances ASC-mediated cell death. PK-1 cells were infected with adenovirus-expressing ASC or GFP (control). Twenty-four hours after infection, the cells were incubated with or without hypoxia and were subsequently collected at the indicated times to undergo both Western blotting and Trypan blue staining analyses.

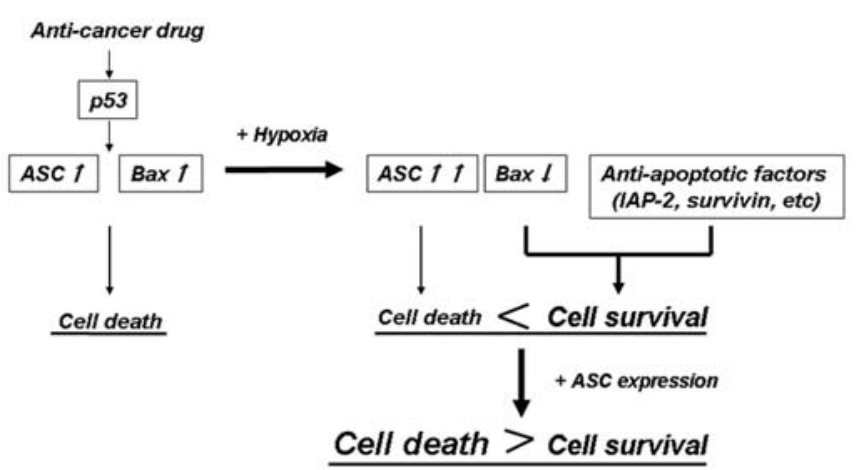

Figure 5. The possible mechanism of hypoxia-induced chemoresistance and the effect of ASC on hypoxia-mediated cell death in pancreatic cancer cells. using Trypan blue exclusion analysis. Fig. 4 showed that the expressed ASC induced $20 \%$ cell death even under normoxia, and that hypoxia increased this ASC-induced cell death to $>40 \% 4$ days after hypoxic incubation.

\section{Discussion}

In the present study, we found ASC to be a novel hypoxiainducible gene expressed in a HIF- $1 \alpha$-dependent manner. Since the protein level of Bax, a collaborator of ASC, decreases under $1 \% \mathrm{O}_{2}$ hypoxia, a physiological level of ASC might not be able to promote cell death. However, ectopically-expressed ASC induced cell death in pancreatic cancer cells and was enhanced by hypoxia. Fig. 5 shows the possible mechanism controlling the role of ASC under hypoxia-mediated cell death. 
Our previous study demonstrated that ASC binds to Bax at the pyrin domain and regulates the translocation of Bax to the mitochondria for the release of cytochrome $\mathrm{c}$, which is a critical step in the intrinsic apoptotic pathway (10). Bax is also a direct target gene of p53, like ASC, thus p53-mediated inductions of ASC and Bax in response to chemotherapeutic drugs are essential elements of mitochondrial cell death signaling $(10,13,14)$. Notably, our present study showed differential regulation of these two genes under $1 \% \mathrm{O}_{2}$ hypoxia: ASC induction and Bax repression. Yokoi and Fidler demonstrated that hypoxia increases the resistance of pancreatic cancer to GEM-induced cell death through the activation of the phosphatidylinositol 3'-kinase (PI3K)/Akt/NF- $\mathrm{KB}$ signaling pathway (6). They also showed that the simultaneous inhibition of growth factor-associated receptors, such as epidermal growth factor receptor (EGFR), vascular endothelial growth factor receptor and platelet-derived growth factor receptor (PDGFR), increases the chemosensitivity of pancreatic cancer to GEM (7). In addition to the contribution of growth factors to chemoresistance in pancreatic cancer, other researchers have shown the effect of apoptosis-related genes on hypoxiamediated chemoresistance or tumor growth using various types of cancer. Liu et al demonstrated the relationship between hypoxia-induced chemoresistance in gastric cancer and $\mathrm{Bcl}-2$ family proteins. In that study, the expression of antiapoptotic gene Bcl-2 and the suppression of pro-apoptotic Bax were possible causes (15). Fei et al also showed the suppression of Bax under hypoxia in sarcoma cells (16). These findings support our observations, and Bax repression might be one of the critical reasons for hypoxia-induced chemoresistance in pancreatic cancer.

Although p53 is well known to be a tumor suppressor gene $(13,14)$, it also induces pro-apoptotic as well as antiapoptotic genes to regulate the balance of cellular death in response to anti-cancer drugs $(17,18)$. It has also been reported that HIF-1 $\alpha$ induces both pro-apoptotic (BNIP3, NOXA, NIX, RTP801) and anti-apoptotic (FOXO3, IAP-2 and survivin) genes (19-23). For each cellular phenotype, the death or survival induced by hypoxia seems to depend on degree. As used in this study, $1 \% \mathrm{O}_{2}$ hypoxia did not promote cell death in pancreatic cancer cells by itself, while others have shown that severe hypoxia $\left(0.1 \% \mathrm{O}_{2}\right)$ or anoxia induced cell death (24). In addition, $1 \% \mathrm{O}_{2}$ hypoxia induces both pro- and antiapoptotic genes; the total balance, however, seems to be antiapoptotic dominant (Fig. 5). One of the reasons for the negative contribution of the physiological level of ASC to cellular death under $1 \% \mathrm{O}_{2}$ hypoxia might be a loss of Bax. As a result, the role of ASC under hypoxia remains unclear. It is also unknown whether ASC is a direct target gene of transcriptional factor HIF-1 $\alpha$. ASC might have a greater capacity for cellular death under severely hypoxic conditions, thus further investigation is required to clarify its precise role under hypoxia.

To overcome this anti-apoptotic-dominant condition under hypoxia, we infected adenovirus-expressing ASC into pancreatic cancer cells. ASC gene induction successfully promoted cell death in pancreatic cancer cells even under normoxia, and was enhanced by $1 \% \mathrm{O}_{2}$ hypoxia (Figs. 4 and 5). There are two ASC-associated death signaling pathways; namely, the p53-dependent Bax-mitochondrial pathway and the p53-independent death receptor-caspase 8 pathway (10). The latter seems to be the main pathway of ASC-mediated cell death under hypoxia, since hypoxia causes Bax repression (Fig. 3). ASC has 2 protein adaptor domains, and therefore has the potential to bind to various types of molecules, regulating cellular death in response to various death stimuli (9-12). Since there have been no reports describing the relationship between ASC and hypoxia, subsequent study should identify the hypoxia-inducible genes which collaborate with ASC under hypoxia. In any event, the findings of the current study suggest that ASC gene induction is a potential new treatment strategy for pancreatic cancer.

\section{Acknowledgements}

This study was supported by a Grant-in-Aid for Scientific Research from the Ministry of Education, Science, Sports, and Culture of Japan (no.19790943).

\section{References}

1. Warshaw AL and Fernandez-del Castillo C: Pancreatic carcinoma. N Engl J Med 326: 455-465, 1992.

2. Koong AC, Metha VK, Le QT, et al: Pancreatic tumors show high levels of hypoxia. Int J Radiat Oncol Biol Phys 48: 919-922, 2000.

3. Maxwell PH, Dachs GU, Gladle JM, et al: Hypoxia-inducible factor-1 modulates gene expression in solid tumors and influences both angiogenesis and tumor growth. Proc Natl Acad Sci USA 94: 8104-8109, 1997

4. Ide T, Kitajima Y, Miyoshi A, et al: Tumor-stromal interaction under hypoxia increases the invasiveness of pancreatic cancer cells through the hepatocyte growth factor/c-Met pathway. Int $\mathbf{J}$ Cancer 119: 2750-2759, 2006.

5. Ide T, Kitajima Y, Miyoshi A, et al: The hypoxic environment in tumor-stromal cells accelerates pancreatic cancer progression via the activation of paracrine hepatocyte growth factor/c-Met signaling. Ann Surg Oncol 14: 2600-2607, 2007.

6. Yokoi K and Fidler IJ: Hypoxia increases resistance to human pancreatic cancer cells to apoptosis induced by gemcitabine. Clin Cancer Res 10: 2299-2306, 2004.

7. Yokoi K, Sasaki T, Bucana CD, et al: Simultaneous inhibition of EGFR, VEGFR, and platelet-derived growth factor receptor signaling combined with gemcitabine produces therapy of human pancreatic carcinoma and prolongs survival in an orthotopic nude mouse model. Cancer Res 65: 10371-10380, 2005

8. Chang Q, Qin R, Huang T, Gao J and Feng Y: Effect of antisense hypoxia-inducible factor $1 \alpha$ on progression, metastasis and chemosensitivity of pancreatic cancer. Pancreas 32: 297-305, 2006.

9. Masumoto J, Taniguchi S, Ayukawa K, et al: ASC, a novel 22-kDa protein, aggregates during apoptosis of human promyelocytic leukemia HL-60 cells. J Biol Chem 274: 33825-33838, 1999.

10. Ohtsuka T, Ryu H, Minamishima YA, et al: ASC is a Bax adaptor and regulates the p53-Bax mitochondrial apoptosis pathway. Nat Cell Biol 6: 121-128, 2004.

11. Ohtsuka T, Liu XF, Koga Y, et al: Methylation-induced silencing of ASC and the effect of expressed ASC on p53-mediated chemosensitivity in colorectal cancer. Oncogene 25: 1807-1811, 2006.

12. Stehlik C, Fiorentino L, Dorfleutner A, et al: The PAAD/ PYRIN-family protein ASC is a dual regulator of a conserved step in nuclear factor $\kappa \mathrm{B}$ activation pathway. J Exp Med 196: 1605-1615, 2002.

13. Vogelstein B, Lane D and Levine AJ: Surfing the p53 network. Nature 408: 307-310, 2000.

14. Gross A, McDonnell JM and Korsmeyer SJ: BCL-2 family members and the mitochondria in apoptosis. Genes Dev 13: 1899-1911, 1999

15. Liu L, Ning X, Sun L, et al: Hypoxia-inducible factor- $1 \alpha$ contributes to hypoxia-induced chemoresistance in gastric cancer. Cancer Sci 99: 121-128, 2008.

16. Fei P, Wang W, Kim SH, et al: Bnip3L is induced by p53 under hypoxia, and its knockdown promotes tumor growth. Cancer Cell 6: 597-609, 2004. 
17. Han JA, Kim JL, Ongusaha PP, et al: p53-mediated induction of Cox-2 counteracts p53- or genotoxic stress-induced apoptosis. EMBO J 21: 5635-5644, 2002.

18. Ongusaha PP, Kim JL, Fang L, et al: p53 induction and activation of DDR1 kinase counteract p53-mediated apoptosis and influence p53 regulation through a positive feedback loop. EMBO J 22: 1289-1301, 2003.

19. Kim JY, Ahn HJ, Ryu JH, Suk K and Park JH: BH3-only protein Noxa is a mediator of hypoxic cell death induced by hypoxiainducible factor 1 $\alpha$. J Exp Med 199: 113-123, 2004.

20. Schmid T, Zhou J and Brue B: HIF-1 and p53: communication of transcription factors under hypoxia. J Cell Mol Med 4: 423-431, 2004.
21. Akada M, Crnogorac-Jurcevic T, Lattimore S, et al: Intrinsic chemoresistance to gemcitabine is associated with decreased expression of Bnip3 in pancreatic cancer. Clin Cancer Res 11: 3094-3101, 2005.

22. Okami J, Simeone DM and Logsdon CD: Silencing of the hypoxia-inducible cell death protein in pancreatic cancer. Cancer Res 64: 5338-5346, 2004.

23. Bakker WJ, Harris IS and Mak TW: FOXO3a is activated in response to hypoxic stress and inhibits HIF1-induced apoptosis via regulation of CITED2. Mol Cell 28: 941-953, 2007.

24. Yasuda M, Matsubara J, Yamasaki H, et al: Death-resistant and nonresistant malignant human cell lines under anoxia in vitro. Int J Clin Oncol 12: 455-462, 2007. 